\title{
Introduction to the special issue on numerical simulation of optoelectronic devices NUSOD'14
}

\author{
Weida $\mathrm{Hu}^{1} \cdot$ Eugene Avrutin $^{2} \cdot$ Julien Javaloyes $^{3}$ • \\ Sławomir Sujecki ${ }^{4} \cdot$ Mohamed Swillam $^{5}$
}

Published online: 9 April 2015

(C) Springer Science+Business Media New York 2015

This special issue of Optical and Quantum Electronics contains a selection of papers that were presented at the 14th International Conference on Numerical Simulation of Optoelectronic Devices (NUSOD) in Mallorca, Spain, September 1-4 2014. NUSOD'14 was chaired by Julien Javaloyes from the University of the Balearic Islands, Spain and Joachim Piprek from NUSOD Institute, USA.

The purpose of NUSOD Conference is to provide a forum for scientists from research labs around the world to discuss the recent advances in the modelling of optoelectronic devices, materials, and applications. This year the main topics included numerical modelling of semiconductor lasers, light emitting diodes, optical modulators, optical amplifiers, photodetectors, solar cells, photonic devices and circuits, optoelectronic integrated circuits.

Weida $\mathrm{Hu}$

wdhu@mail.sitp.ac.cn

Eugene Avrutin

eugene.avrutin@york.ac.uk

Julien Javaloyes

julien.javaloyes@uib.es

Sławomir Sujecki

slawomir.sujecki@nottingham.ac.uk

Mohamed Swillam

mohamed.swillam@gmail.com

1 National Lab for Infrared Physics, Shanghai Institute of Technical Physics, Chinese Academy of Sciences, Shanghai 200083, China

2 Department of Electronics, University of York, York, UK

3 Department of Physics, University of the Balearic Islands, Palma, Spain

4 Department of Electrical and Electronic Engineering, University of Nottingham, Nottingham, UK

5 Department of Physics, American University of Cairo, Cairo, Egypt 
The papers collected in this special issue of Optical and Quantum Electronics provide a comprehensive representation of the topics discussed at the conference.

The editor team would like to thank all the authors who submitted their papers to the special issue and the reviewers for their work and timely response. We now look forward to NUSOD'15 that will take place in Taipei, Taiwan, September 7-11, 2015. 